Research Article

\title{
Further Properties of Trees with Minimal Atom-Bond Connectivity Index
}

\author{
Jianping Liu and Jinsong Chen \\ College of Mathematics and Computer Science, Fuzhou University, Fuzhou 350116, China \\ Correspondence should be addressed to Jinsong Chen; fzu_cjs@126.com
}

Received 22 April 2014; Accepted 27 May 2014; Published 19 June 2014

Academic Editor: Yonghui Xia

Copyright ( 92014 J. Liu and J. Chen. This is an open access article distributed under the Creative Commons Attribution License, which permits unrestricted use, distribution, and reproduction in any medium, provided the original work is properly cited.

Let $G=(V, E)$ be a graph the atom-bond connectivity $(\mathrm{ABC})$ index is defined as the sum of weights $\left(\left(d_{u}+d_{v}-2\right) / d_{u} d_{v}\right)^{1 / 2}$ over all edges $u v$ of $G$, where $d_{u}$ denotes the degree of a vertex $u$ of $G$. In this paper, we determined a few structural features of the trees with minimal $\mathrm{ABC}$ index also we characterized the trees with $\operatorname{dia}[T]=2$ and minimal $\mathrm{ABC}$ index, where $[T]$ is induced by the vertices of degree greater than 2 in $T$ and $\operatorname{dia}[T]$ is the diameter of $[T]$.

\section{Introduction}

Let $G=(V, E)$ be a finite, simple, and undirected graph. The degree of a vertex $u \in V$ is denoted by $d_{u}$. The atom-bond connectivity $(\mathrm{ABC})$ index is defined as the sum of weights $\left(\left(d_{u}+d_{v}-2\right) / d_{u} d_{v}\right)^{1 / 2}$ over all edges $u v$ of $G$; that is,

$$
\operatorname{ABC}(G)=\sum_{u v \in E(G)} \sqrt{\frac{d_{u}+d_{v}-2}{d_{u} d_{v}}} .
$$

The ABC index of a graph was defined by Estrada et al. [1] and it has many chemical applications $[1,2]$.

When examining a topological index, one of the fundamental questions that needs to be answered is for which graphs this index assumes minimal and maximal values and what are these extremal values. In the case of the $A B C$ index, finding the tree for which this index is maximal was relatively easy [3]; it is the star. Eventually, also the trees with second-maximal, third-maximal, and so forth $\mathrm{ABC}$ index were determined [4].

We [5] have shown that by deleting an edge from any graph, the ABC index decreases. This result implies that among all $n$-vertex graphs, the complete graph $K_{n}$ has maximal $\mathrm{ABC}$ value. Further, among all connected $n$-vertex graphs, minimal ABC is achieved by some tree. Thus the $n$ vertex trees with minimal $\mathrm{ABC}$ index are also the $n$-vertex connected graphs with minimal $\mathrm{ABC}$ index. But the problem of characterizing the $n$-vertex trees with minimal ABC index turned out to be much more difficult, and a complete solution of this problem is not known. For more results on $\mathrm{ABC}$ index see [6-13].

In a recent work [6] a combination of computer search and mathematical analysis was undertaken, aimed at elucidating the structure of the minimal $\mathrm{ABC}$ trees. And some structural features of the trees with minimal $A B C$ index are given in [7].

Lemma 1 (see [6]). If $n \geq 10$, then the $n$-vertex tree with minimal $A B C$ index contains at most one pendent path of length $k=3$.

Lemma 2 (see [7]). If $n \geq 10$, then each pendent vertex of the $n$-vertex tree $G$ with minimal $A B C$ index belongs to a pendent path of length $k, 2 \leq k \leq 3$.

By inspecting the structural features of these trees, in [8] the branches $B_{1}, \ldots, B_{5}$ and $B_{3}^{*}$ were given. Let $B_{i}$ be a branch of tree $T$ formed by attaching $i$ pendant path of length 2 to the vertex $v$ such that the degree of $v$ in $T$ is $i+1$. Let $B_{i}^{*}$ be a branch of tree $T$ formed by attaching $i-1$ pendant path of length 2 and a pendant path of length 3 to the vertex $v$ such that the degree of $v$ in $T$ is $i+1$ (see Figure 1). Denote by $k B_{i}$ the $k$ union of the branches $B_{i}$ and by $N\left(B_{i}\right)$ the number of 


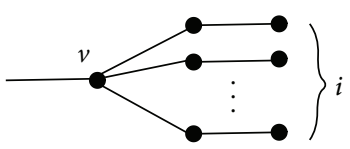

$B_{i}$

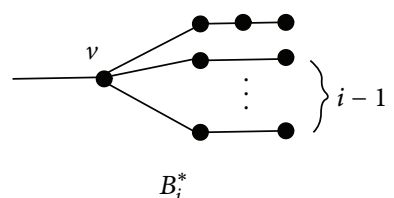

$B_{i}^{*}$
Figure 1: The branches $B_{i}$ and $B_{i}^{*}$.

branches $B_{i}$ in $T$. From Lemmas 1 and 2 , we know all branches in a tree $T$ with minimal ABC index must be of the type $B_{i}$ or $B_{i}^{*}$, and $N\left(B_{i}^{*}\right) \leq 1, i=1,2, \ldots$. According to Lemma 1 , in the following we assume that $N\left(B_{3}^{*}\right) \leq 1$ and $N\left(B_{i}^{*}\right)=0$, for all $i \neq 3$.

In [9] the $n$-vertex minimal ABC trees were determined up to $n=300$ and then a conjecture about the trees with minimal $\mathrm{ABC}$ index was presented.

Conjecture 3 (see [9]). Let $G$ be a tree with minimal $A B C$ index among all trees of size $n$. Let $T_{0}, T_{1}, T_{2}, T_{3}, T_{4}, T_{5}$, and $T_{6}$ be the structures depicted in Figure 2.

(i) If $n \equiv 0(\bmod 7), n \geq 175$ and $n=7 k+28$, then $G$ has the structure $T_{0}$.

(ii) If $n \equiv 1(\bmod 7), n \geq 64$ and $n=7 k+1$, then $G$ has the structure $T_{1}$.

(iii) If $n \equiv 2(\bmod 7), n \geq 1185$ and $n=7 k+9$, then $G$ has the structure $T_{2}$.

(iv) If $n \equiv 3(\bmod 7), n \geq 80$ and $n=7 k+10$, then $G$ has the structure $T_{3}$.

(v) If $n \equiv 4(\bmod 7), n \geq 312$ and $n=7 k+11$, then $G$ has the structure $T_{4}$.

(vi) If $n \equiv 5(\bmod 7), n \geq 117$ and $n=7 k+19$, then $G$ has the structure $T_{5}$.

(vii) If $n \equiv 6(\bmod 7), n \geq 62$ and $n=7 k+6$, then $G$ has the structure $T_{6}$.

In this paper, we determined a few structural features of the trees with minimal $\mathrm{ABC}$ index, also we characterized the trees with $\operatorname{dia}[T]=2$ and minimal $\mathrm{ABC}$ index, where $\operatorname{dia}[T]$ is the diameter of $[T]$, which was induced by the vertices of degree greater than 2 in $T$.

\section{The Structural Features of the Trees with Minimal ABC Index}

Now, we are going to determine a few structural features of the trees with minimal $\mathrm{ABC}$ index.

Theorem 4. The n-vertex tree with minimal $A B C$ index does not contain branches $B_{k}$ and $B_{k}^{*}(k \geq 6)$.

Proof. Suppose that $T_{k}^{1}$ is a tree with minimal ABC index, possessing a branch $B_{k}, k \geq 6$. Let $u$ be a vertex of $T_{k}^{1}$, adjacent to the vertex $v$, and the degree of $u$ is $s$. Consider the tree $T_{k}^{2}$ (see Figure 3).
By direct calculation, we have

$$
\begin{aligned}
& \operatorname{ABC}\left(T_{k}^{1}\right)-\operatorname{ABC}\left(T_{k}^{2}\right) \\
& =\sqrt{\frac{k+s-1}{(k+1) s}}+\sqrt{2}-\sqrt{\frac{k+s-4}{(k-2) s}}-2 \sqrt{\frac{k-1}{3(k-2)}} .
\end{aligned}
$$

If $k=6$, it can be easily checked by computer that

$$
\begin{aligned}
& \sqrt{\frac{s+5}{7 s}}+\sqrt{2}-\sqrt{\frac{s+2}{4 s}}-2 \sqrt{\frac{5}{12}}>0, \\
& \text { that is, } \operatorname{ABC}\left(T_{k}^{1}\right)>\operatorname{ABC}\left(T_{k}^{2}\right) .
\end{aligned}
$$

For the case $k \geq 7$, if the inequality $\operatorname{ABC}\left(T_{k}^{1}\right)>\operatorname{ABC}\left(T_{k}^{2}\right)$ holds, it implies that

$$
\begin{gathered}
\frac{k+s-1}{(k+1) s}+2+2 \sqrt{\frac{2 k+2 s-2}{(k+1) s}} \\
>\frac{k+s-4}{(k-2) s}+\frac{4(k-1)}{3(k-2)} \\
+\frac{4 \sqrt{(k-1)(k+s-4)}}{(k-2) \sqrt{3 s}} .
\end{gathered}
$$

By elementary calculation, this inequality can be transformed to

$$
\begin{aligned}
3(k-2) & (k+s-1)+6 s(k+1)(k-2) \\
& -3(k+1)(k+s-4)-4 s(k+1)(k-1) \\
> & 4(k+1) \sqrt{3 s(k-1)(k+s-4)} \\
& -6(k-2) \sqrt{s(k+1)(2 k+2 s-2)} .
\end{aligned}
$$

That is,

$$
\begin{aligned}
2 k^{2} s & -6 k s-17 s+18+6(k-2) \\
& \times \sqrt{s(k+1)(2 k+2 s-2)} \\
> & 4(k+1) \sqrt{3 s(k-1)(k+s-4)} .
\end{aligned}
$$

By squaring the above relation and rearranging, we get

$$
\begin{aligned}
& \left(4 k^{4} s^{2}-196 k^{2} s^{2}\right)+\left(252 k s^{2}-1092 s\right) \\
& +\left(24 k^{4} s-144 k^{3} s\right) \\
& +\left(528 k^{2} s-72 k s\right)+625 s^{2}+324 \\
& +\left(12 \sqrt{2}(k-2)\left(2 k^{2} s-6 k s-17 s+18\right)\right. \\
& \left.\quad \times \sqrt{(k+1) s(k+s-1)}-100 k^{2} s^{2}\right)>0 .
\end{aligned}
$$



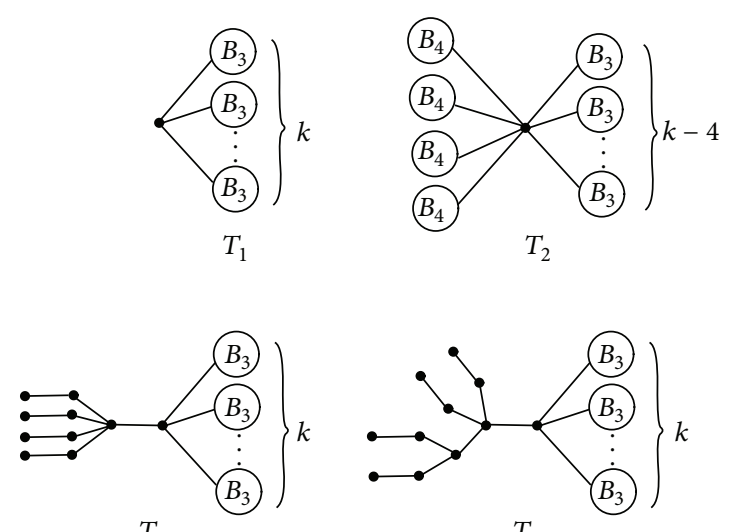

$T_{3}$

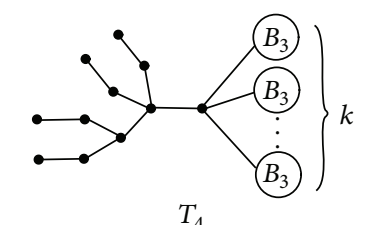

$T_{4}$

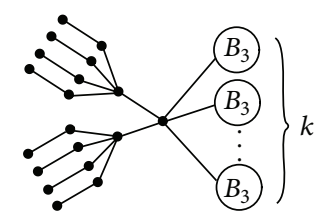

$T_{5}$

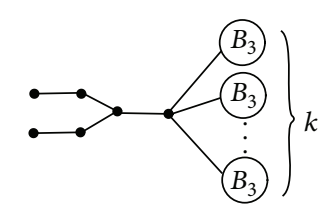

$T_{6}$

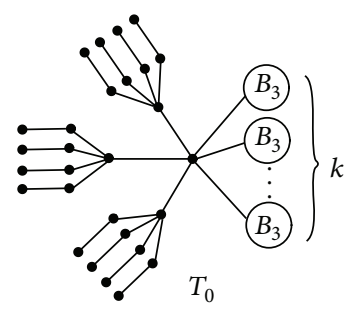

FIgUre 2: Types of trees with minimal ABC index correspond to Conjecture 3.

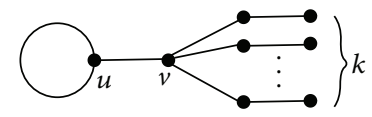

$T_{k}^{1}$

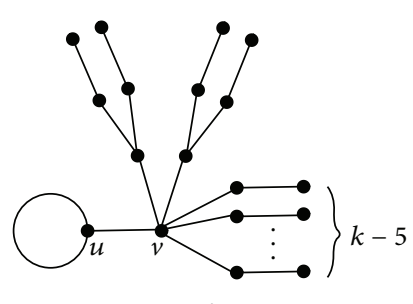

$T_{k}^{2}$

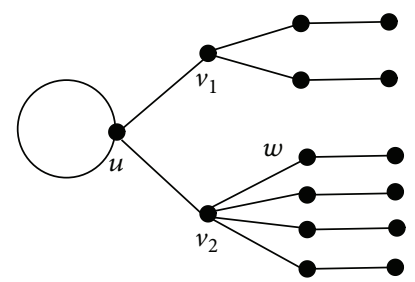

$T^{1}$

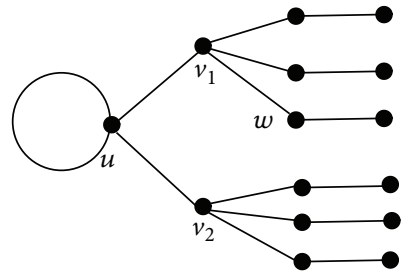

$T^{2}$

FIgURE 3

FIGURE 4

Since the function

$$
\begin{aligned}
& g(k, s) \\
& =12 \sqrt{2}(k-2)\left(2 k^{2} s-6 k s-17 s+18\right) \\
& \quad \times \sqrt{(k+1) s(k+s-1)}-100 k^{2} s^{2} . \\
& >16(k-2)\left(2 k^{2}-6 k-17\right) \\
& \quad \times \sqrt{k+1} s^{2}-100 k^{2} s^{2}>0 \quad(\text { as } k \geq 7, s \geq 3),
\end{aligned}
$$

and it holds that

$$
\begin{aligned}
& 4 k^{4} s^{2}-196 k^{2} s^{2}>0, \\
& 252 k s^{2}-1092 s>0, \\
& 24 k^{4} s-144 k^{3} s>0, \\
& 528 k^{2} s-72 k s>0 \quad(\text { as } k \geq 7, s \geq 3),
\end{aligned}
$$

thus, we have

$$
\operatorname{ABC}\left(T_{k}^{1}\right)>\operatorname{ABC}\left(T_{k}^{2}\right), \quad \text { for } k \geq 7
$$

In the same way, we can prove that the $n$-vertex tree with minimal ABC index does not contain branch $B_{k}^{*}(k \geq 6)$.

The proof is complete.

Note that Theorem 4 holds for all $n$-vertex trees with minimal ABC index.

Theorem 5. Let $T$ be a tree with minimal $A B C$ index, then every vertex of $T$ must not be connected with both $B_{2}$ and $B_{4}$.

Proof. Suppose that $T^{1}$ is a tree with minimal ABC index; let $u$ be a vertex of $T^{1}$, which is connected with both $B_{2}$ and $B_{4}$, and the degree of $u$ is $s(s \geq 3)$. Construct the tree $T^{2}$ by deleting the edge $v_{2} w$ and connecting $w$ with $v_{1}$ (see Figure 4 ). 
The transformation $T^{1} \rightarrow T^{2}$ causes the following change of the $A B C$ index:

$$
\mathrm{ABC}\left(T^{1}\right)-\mathrm{ABC}\left(T^{2}\right)=\sqrt{\frac{s+3}{5 s}}+\sqrt{\frac{s+1}{3 s}}-2 \sqrt{\frac{s+2}{4 s}} .
$$

If the inequality $\mathrm{ABC}\left(T^{1}\right)>\mathrm{ABC}\left(T^{2}\right)$ holds, it implies that

$$
\frac{s+3}{5 s}+\frac{s+1}{3 s}+2 \sqrt{\frac{(s+1)(s+3)}{15 s^{2}}}>\frac{s+2}{s} .
$$

By elementary calculation, this inequality can be transformed to

$$
11 s^{2}+16 s-76>0 \quad(s \geq 3) .
$$

Thus we have $\mathrm{ABC}\left(T^{1}\right)>\mathrm{ABC}\left(T^{2}\right)$, for $s \geq 3$.

The proof is complete.

Theorem 6. Let $T$ be a tree with minimal $A B C$ index; then every vertex of $T$ must not be connected with both $B_{1}$ and $2 B_{4}$.

Proof. Suppose that $T^{3}$ is a tree with minimal ABC index; let $u$ be a vertex of $T^{3}$, which is connected with both $B_{1}$ and $2 B_{4}$, and the degree of $u$ is $s$ (obviously $s \geq 3$ ). Construct the tree $T^{4}$ by deleting the edges $v_{2} w_{2}, v_{3} w_{3}$ and adding the edges $w_{1} w_{2}, v_{1} w_{3}$ (see Figure 5).

The transformation $T^{3} \rightarrow T^{4}$ causes the following change of the $A B C$ index:

$$
\begin{aligned}
& \operatorname{ABC}\left(T^{3}\right)-\operatorname{ABC}\left(T^{4}\right) \\
& =\frac{\sqrt{2}}{2}+2 \sqrt{\frac{s+3}{5 s}}-\sqrt{\frac{s+1}{3 s}}-2 \sqrt{\frac{s+2}{4 s}} .
\end{aligned}
$$

If the inequality $\mathrm{ABC}\left(\mathrm{T}^{3}\right)>\mathrm{ABC}\left(\mathrm{T}^{4}\right)$ holds, it implies that

$$
\frac{1}{2}+\frac{4(s+3)}{5 s}+2 \sqrt{\frac{2(s+3)}{5 s}}>\frac{4 s+7}{3 s}+\frac{2}{s} \sqrt{\frac{s^{2}+3 s+2}{3}} .
$$

That is

$$
\frac{1}{2}+\frac{4(s+3)}{5 s}+2 \sqrt{\frac{2(s+3)}{5 s}}-\frac{4 s+7}{3 s}>\frac{2}{s} \sqrt{\frac{s^{2}+3 s+2}{3}} .
$$

By squaring the above relation and rearranging, we get

$$
\frac{(s-2)(1198+(241-24 \sqrt{(10 s+30) / s}) s)}{900 s^{2}}>0 \quad(s \geq 3) .
$$

Thus we have $\mathrm{ABC}\left(T^{3}\right)>\operatorname{ABC}\left(T^{4}\right)$, for $s \geq 3$, and the proof is complete.

Theorem 7. Let $T$ be a tree with minimal $A B C$ index; then every vertex of $T$ must not be connected with $7 B_{4}$.

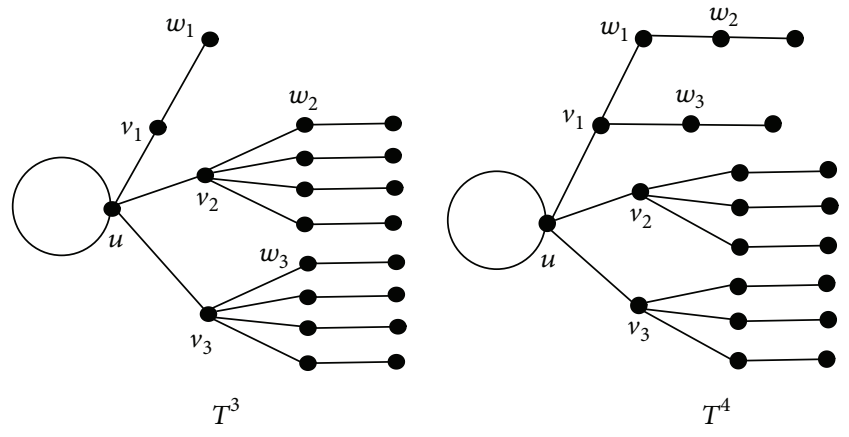

FIGURE 5

Proof. Suppose that $T^{7}$ is a tree with minimal ABC index, possessing a vertex $u$ in $T^{7}$ connected with $7 B_{4}$ (see Figure 6). Let $U=\left\{v_{1}, v_{2}, \ldots, v_{s}\right\}$ be the set of adjacent vertices to $u$. Let $d_{1}, d_{2}, \ldots, d_{s}$ be the degree of $v_{1}, v_{2}, \ldots, v_{s}$, respectively. We consider the tree $T^{8}$ shown in Figure 6 .

Here we are going to show that $\mathrm{ABC}\left(T^{7}\right)-\mathrm{ABC}\left(T^{8}\right)>0$, for any $s \geq 7$.

Consider

$$
\begin{aligned}
\operatorname{ABC}( & \left.T^{7}\right)-\operatorname{ABC}\left(T^{8}\right) \\
= & 7 \sqrt{\frac{s+3}{5 s}}+\sqrt{2}-9 \sqrt{\frac{s+4}{4(s+2)}} \\
& +\sum_{i=8}^{s}\left(\sqrt{\frac{s+d_{i}-2}{s d_{i}}}-\sqrt{\frac{s+d_{i}}{(s+2) d_{i}}}\right),
\end{aligned}
$$

for any $d_{i} \geq 2, \sqrt{\left(s+d_{i}-2\right) /\left(s d_{i}\right)}-\sqrt{\left(s+d_{i}\right) /\left((s+2) d_{i}\right)}>0$.

Now we are going to show that $7 \sqrt{(s+3) /(5 s)}+\sqrt{2}>$ $9 \sqrt{(s+4) / 4(s+2)}$; that is

$$
\frac{49(s+3)}{5 s}+2+14 \sqrt{\frac{2(s+3)}{5 s}}>\frac{81(s+4)}{4(s+2)} .
$$

By elementary calculation, this inequality can be transformed to

$$
2799 s^{4}+30240 s^{3}+585648 s^{2}+1693440 s-1382976>0 .
$$

The largest root of the above polynomial is 0.660387 ; therefore, the value of the above polynomial is positive for $s>0.660387$.

Thus we have $\operatorname{ABC}\left(T^{7}\right)>\operatorname{ABC}\left(T^{8}\right)$, and the proof is complete.

Theorem 8. Let $T$ be a tree with minimal $A B C$ index; then every vertex of $T$ must not be connected with $2 B_{5}$.

Proof. Suppose that $T_{5}^{1}$ is a tree with minimal ABC index, possessing a vertex connected with $2 B_{5}$. Let $u$ be the vertex of $T_{5}^{1}$, adjacent to the vertices $v_{1}$ and $v_{2}$, and the degree of $u$ is $s\left(s \geq 3\right.$ ). Consider the tree $T_{5}^{2}$ (see Figure 7). 


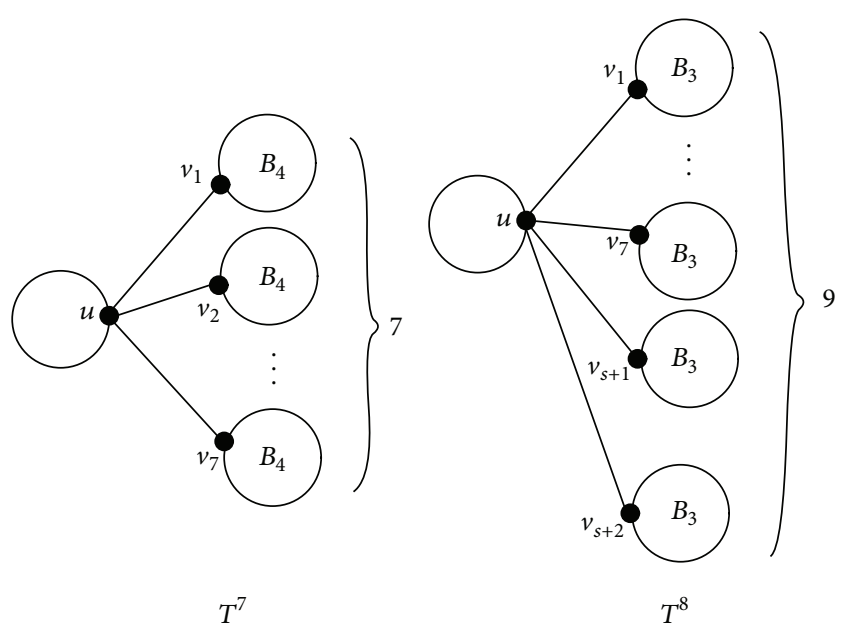

FIGURE 6

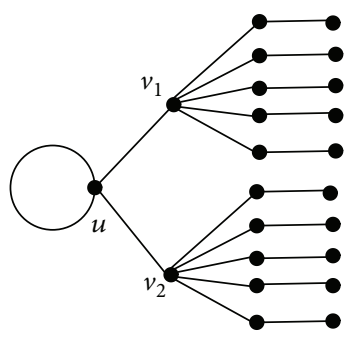

$T_{5}^{1}$

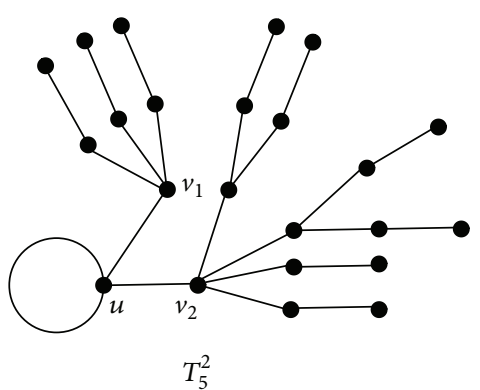

Figure 7
The transformation $T_{5}^{1} \rightarrow T_{5}^{2}$ causes the following change of the $\mathrm{ABC}$ index:

$$
\begin{aligned}
\operatorname{ABC} & \left(T_{5}^{1}\right)-\operatorname{ABC}\left(T_{5}^{2}\right) \\
& =2 \sqrt{\frac{s+4}{6 s}}+\sqrt{2}-\sqrt{\frac{s+2}{4 s}}-\sqrt{\frac{s+3}{5 s}}-2 \sqrt{\frac{6}{15}} .
\end{aligned}
$$

It can be easily checked by computer that $\operatorname{ABC}\left(T_{5}^{1}\right)>$ $\operatorname{ABC}\left(T_{5}^{2}\right)$, for $s \geq 3$.

The proof is complete.

\section{The Minimal ABC Indices of Trees with Order $n$ and $\operatorname{dia}[T]=2$}

Denote by $[T]$ the subgraph of $T$ induced by its vertices of degree greater than 2 . For a connected graph $G$, the diameter of $G$, denoted by $\operatorname{dia} G$, is the length of a longest path of $G$.

Lemma 9 (see [7]). Let $T$ be an $n$-vertex $(n \geq 10)$ tree with minimal $A B C$ index; then $[T]$ is a tree.

Note that the structures $T_{i}$ in Conjecture 3 have dia $\left[T_{i}\right]=$ $2(i=0,1,2,3,5,6)$. Let $T_{n, 2}$ be the set of $n$-vertex trees $T$ with $\operatorname{dia}[T]=2$.

Now we will characterize the trees with $\operatorname{dia}[T]=2$ and minimal ABC index, which partially solve Conjecture 3.

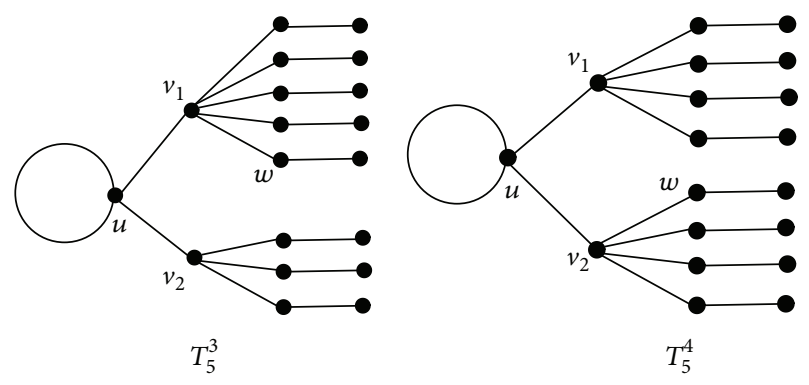

FiguRE 8

Lemma 10. Let $T \in T_{n, 2}$ be a tree with minimal $A B C$ index; then $T$ does not contain $B_{5}$.

Proof. Let $T_{5}^{3} \in T_{n, 2}$ be a tree with minimal ABC index. By Theorems 7 and $8, T_{5}^{3}$ contains at most $6 B_{4}, 1 B_{5}$; hence, for $n>65, T_{5}^{3}$ must contain $B_{3}$. Suppose that $T_{5}^{3}$ possesses $B_{5}$; then we can construct the tree $T_{5}^{4}$ by deleting the edge $v_{1} w$ and adding the edge $v_{2} w$ (see Figure 8).

The transformation $T_{5}^{3} \rightarrow T_{5}^{4}$ causes the following change of the $\mathrm{ABC}$ index:

$$
\begin{gathered}
\operatorname{ABC}\left(T_{5}^{3}\right)-\operatorname{ABC}\left(T_{5}^{4}\right) \\
=\sqrt{\frac{d_{u}+4}{6 d_{u}}}+\sqrt{\frac{d_{u}+2}{4 d_{u}}}-2 \sqrt{\frac{d_{u}+3}{5 d_{u}}} .
\end{gathered}
$$

It can be easily checked that $\mathrm{ABC}\left(T_{5}^{3}\right)>\operatorname{ABC}\left(T_{5}^{4}\right)$, for $d_{u} \geq 3$.

The proof is complete.

Lemma 11. Let $T \in T_{n, 2}$ be a tree with minimal $A B C$ index; if the maximum degree $\Delta \geq 24$, then $T$ must not contain $k B_{2}$ $(k \geq 3)$.

Proof. Suppose that $T^{5} \in T_{n, 2}$ is a tree with minimal ABC index, possessing $3 B_{2}$ (see Figure 9 ). Let $u$ be the vertex with maximum degree of $T^{5}$, adjacent to the vertices $v_{1}, v_{2}$, and $v_{3}$, and the degree of $u$ is $s+1$. Construct the tree $T^{6}$ by deleting the edges $v_{3} w_{1}$ and $u v_{3}$ and adding the edges $v_{1} w_{1}$ and $v_{2} v_{3}$. Let $U=\left\{v_{1}, v_{2}, \ldots, v_{s+1}\right\}$ be a set of adjacent vertices to $u$. Let $d_{1}, d_{2}, \ldots, d_{s+1}$ be the degree of $v_{1}, v_{2}, \ldots, v_{s+1}$, respectively.

The transformation $T^{5} \rightarrow T^{6}$ causes the following change of the $\mathrm{ABC}$ index:

$$
\begin{aligned}
\operatorname{ABC} & \left(T^{5}\right)-\operatorname{ABC}\left(T^{6}\right) \\
= & 3 \sqrt{\frac{s+2}{3(s+1)}}-2 \sqrt{\frac{s+2}{4 s}}-\frac{\sqrt{2}}{2} \\
& +\sum_{i=4}^{s+1}\left(\sqrt{\frac{s+d_{i}-1}{(s+1) d_{i}}}-\sqrt{\frac{s+d_{i}-2}{s d_{i}}}\right) .
\end{aligned}
$$

By Theorems 4 and 5, Lemma 10, and noticing that $T \in T_{n, 2}$, we know that $d_{i}=2,3$ or 4 . 


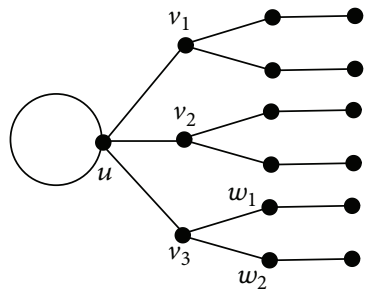

$T^{5}$

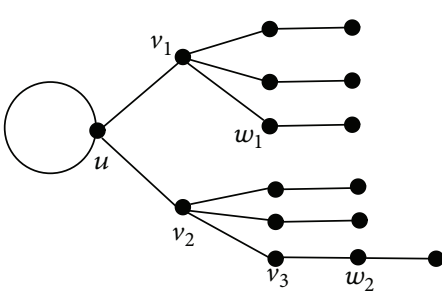

$T^{6}$

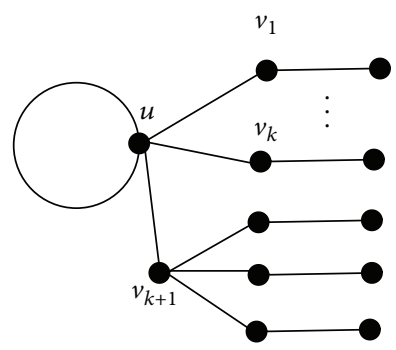

$T^{9}$

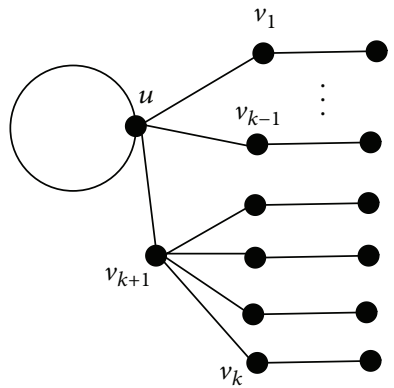

$T^{10}$
Figure 10

And consider

$$
\begin{aligned}
& \sqrt{\frac{s+3}{4(s+1)}}-\sqrt{\frac{s+2}{4 s}} \\
& =\min _{d_{i}=2,3,4}\left(\sqrt{\frac{s+d_{i}-1}{(s+1) d_{i}}}-\sqrt{\frac{s+d_{i}-2}{s d_{i}}}\right) .
\end{aligned}
$$

Putting this in the above expression, we get

$$
\begin{aligned}
\operatorname{ABC} & \left(T^{5}\right)-\operatorname{ABC}\left(T^{6}\right) \\
\geq & 3 \sqrt{\frac{s+2}{3(s+1)}}-2 \sqrt{\frac{s+2}{4 s}}-\frac{\sqrt{2}}{2} \\
& +(s-2)\left(\sqrt{\frac{s+3}{4(s+1)}}-\sqrt{\frac{s+2}{4 s}}\right) \\
= & 3 \sqrt{\frac{s+2}{3(s+1)}}+(s-2) \sqrt{\frac{s+3}{4(s+1)}}-\left(\frac{s}{2} \sqrt{\frac{s+2}{s}}+\frac{\sqrt{2}}{2}\right) .
\end{aligned}
$$

If the inequality $\mathrm{ABC}\left(T^{5}\right)>\operatorname{ABC}\left(T^{6}\right)$ holds, it implies that

$$
\begin{aligned}
& \frac{3(s+2)}{s+1}+\frac{(s-2)^{2}(s+3)}{4(s+1)}+\frac{s-2}{s+1} \sqrt{3(s+2)(s+3)} \\
& >\frac{s(s+2)}{4}+\frac{1}{2}+\sqrt{\frac{s(s+2)}{2}} .
\end{aligned}
$$

By elementary calculation, this inequality can be transformed to

$$
2(s-2) \sqrt{3(s+2)(s+3)}>2 s^{2}-17+(s+1) \sqrt{2 s(s+2)} .
$$

By squaring the above relation and rearranging for two times, we get

$$
\begin{gathered}
s^{8}-20 s^{7}-86 s^{6}+248 s^{5}+956 s^{4}-430 s^{3} \\
-2183 s^{2}-1130 s+\frac{1}{4}>0 .
\end{gathered}
$$

The largest root of the above polynomial is 23.1742; therefore, the value of the above polynomial is positive for $s>23.1742$. Thus we have $\operatorname{ABC}\left(T^{5}\right)>\operatorname{ABC}\left(T^{6}\right)$ for $s \geq 24$, and the proof is complete.

Lemma 12. Let $T \in T_{n, 2}$ be a tree with minimal $A B C$ index; if the maximum degree $\Delta \geq 13$, then $T$ must not contain $B_{1}$.

Proof. Suppose that $T^{9} \in T_{n, 2}$ is a tree with maximum degree $\Delta \geq 13$ and minimal $\mathrm{ABC}$ index, possessing $k B_{1}(k \geq 1)$ (see Figure 10). By Theorem 6 and Lemma 11, we have $N\left(B_{2}\right) \leq 2$ and $N\left(B_{4}\right) \leq 1$. Thus $B_{3}$ in $T^{9}$ must be contained.

Let $u$ be the vertex with maximum degree $s$ in $T^{9}$ and let $U=\left\{v_{1}, v_{2}, \ldots, v_{s}\right\}$ be a set of adjacent vertices to $u$. Let $d_{1}, d_{2}, \ldots, d_{s}$ be the degree of $v_{1}, v_{2}, \ldots, v_{s}$, respectively. Since $T^{9} \in T_{n, 2}$ and by Theorem 4 and Lemma 10, we have $d_{i}=2,3,4$, or 5 . Construct the tree $T^{10}$ by deleting the edge $u v_{k}$ and adding the edge $v_{k+1} v_{k}$ to $T^{9}$.

The transformation $T^{9} \rightarrow T^{10}$ causes the following change of the $\mathrm{ABC}$ index:

$$
\begin{aligned}
\operatorname{ABC} & \left(T^{9}\right)-\operatorname{ABC}\left(T^{10}\right) \\
= & \sum_{i=k+2}^{s}\left(\sqrt{\frac{d_{i}+s-2}{d_{i} s}}-\sqrt{\frac{d_{i}+s-3}{d_{i}(s-1)}}\right) \\
& +\left(\sqrt{\frac{s+2}{4 s}}-\sqrt{\frac{s+2}{5(s-1)}}\right) \\
= & N\left(B_{2}\right)\left(\sqrt{\frac{s+1}{3 s}}-\sqrt{\frac{s}{3(s-1)}}\right) \\
& +N\left(B_{4}\right)\left(\sqrt{\frac{s+3}{5 s}}-\sqrt{\frac{s+2}{5(s-1)}}\right) \\
& +\left(s-k-1-N\left(B_{2}\right)-N\left(B_{4}\right)\right) \\
& \times\left(\sqrt{\frac{s+2}{4 s}}-\sqrt{\frac{s+1}{4(s-1)}}\right)+\left(\sqrt{\frac{s+2}{4 s}}-\sqrt{\frac{s+2}{5(s-1)}}\right) .
\end{aligned}
$$



that

If the inequality $\mathrm{ABC}\left(T^{9}\right)>\mathrm{ABC}\left(T^{10}\right)$ holds, it implies

$$
\begin{aligned}
& \sqrt{\frac{s+2}{4 s}}-\sqrt{\frac{s+2}{5(s-1)}} \\
& >N\left(B_{2}\right)\left(\sqrt{\frac{s}{3(s-1)}}-\sqrt{\frac{s+1}{3 s}}\right) \\
& +N\left(B_{4}\right)\left(\sqrt{\frac{s+2}{5(s-1)}}-\sqrt{\frac{s+3}{5 s}}\right) \\
& +\left(s-k-1-N\left(B_{2}\right)-N\left(B_{4}\right)\right) \\
& \times\left(\sqrt{\frac{s+1}{4(s-1)}}-\sqrt{\frac{s+2}{4 s}}\right) \triangleq f(s, k) .
\end{aligned}
$$

Note that $N\left(B_{2}\right) \leq 2, N\left(B_{4}\right) \leq 1, k \geq 1$, and

$$
\begin{aligned}
\sqrt{\frac{s}{3(s-1)}}-\sqrt{\frac{s+1}{3 s}} & <\sqrt{\frac{s+1}{4(s-1)}}-\sqrt{\frac{s+2}{4 s}} \\
& <\sqrt{\frac{s+2}{5(s-1)}}-\sqrt{\frac{s+3}{5 s}} .
\end{aligned}
$$

We have, $f(s, k) \leq(\sqrt{(s+2) /(5(s-1))}-\sqrt{(s+3) /(5 s)})+(s-$ $3)(\sqrt{(s+1) /(4(s-1))}-\sqrt{(s+2) /(4 s)})$.

Now we are going to show that

$$
\begin{aligned}
\sqrt{\frac{s+2}{4 s}}-\sqrt{\frac{s+2}{5(s-1)}>} & \left(\sqrt{\frac{s+2}{5(s-1)}}-\sqrt{\frac{s+3}{5 s}}\right) \\
& +(s-3)\left(\sqrt{\frac{s+1}{4(s-1)}}-\sqrt{\frac{s+2}{4 s}}\right) .
\end{aligned}
$$

That is,

$$
(s-2) \sqrt{\frac{s+2}{4 s}}-2 \sqrt{\frac{s+2}{5(s-1)}}>(s-3) \sqrt{\frac{s+1}{4(s-1)}}-\sqrt{\frac{s+3}{5 s}} .
$$

By squaring the above relation and rearranging for two times, we get

$$
\begin{aligned}
& 8 \sqrt{5}(s-3)(5 s-4)\left(2 s^{2}-s+7\right) \sqrt{s(s-1)(s+1)(s+3)} \\
& >140 s^{6}+180 s^{5}-2709 s^{4}+1734 s^{3} \\
& \quad+2151 s^{2}-776 s-784
\end{aligned}
$$

Since $\sqrt{s(s-1)(s+1)(s+3)}>(s+0.6)^{2}$ (for $\left.s \geq 7\right)$ and the largest root of the following polynomial is 12.9172 ,

$$
\begin{aligned}
8 \sqrt{5} & (s-3)(5 s-4)\left(2 s^{2}-s+7\right)(s+0.6)^{2} \\
& -140 s^{6}-180 s^{5}+2709 s^{4}-1734 s^{3} \\
& -2151 s^{2}+776 s+784 \\
= & 0 .
\end{aligned}
$$

Therefore the value of the above polynomial is positive for $s>12.9172$. Thus we have $\mathrm{ABC}\left(T^{9}\right)>\operatorname{ABC}\left(T^{10}\right)$ for $s \geq 13$ and the proof is complete.

Theorem 13. Let $T \in T_{n, 2}$ be a tree with minimal $A B C$ index and the maximum degree $\Delta \geq 24$. Let $T_{0}, T_{1}, T_{2}, T_{3}, T_{5}$, and $T_{6}$ be the structures depicted in Figure 2.

(i) If $n \equiv 0(\bmod 7), n \geq 175$ and $n=7 k+28$, then $T$ has the structure $T_{0}$.

(ii) If $n \equiv 1(\bmod 7), n \geq 169$ and $n=7 k+1$, then $T$ has the structure $T_{1}$.

(iii) If $n \equiv 2(\bmod 7), n \geq 1185$ and $n=7 k+9$, then $T$ has the structure $T_{2}$.

(iv) If $n \equiv 3(\bmod 7), n \geq 171$ and $n=7 k+10$, then $T$ has the structure $T_{3}$.

(v) If $n \equiv 4(\bmod 7), n \geq 2020$ and $n=7 k+11$, then $T$ has the structure $T_{4}^{\prime}$ depicted in Figure 11(b).

(vi) If $n \equiv 5(\bmod 7), n \geq 173$ and $n=7 k+19$, then $T$ has the structure $T_{5}$.

(vii) If $n \equiv 6(\bmod 7), n \geq 167$ and $n=7 k+6$, then $T$ has the structure $T_{6}$.

Proof. Let $T \in T_{n, 2}$ be a tree with minimal ABC index. From Lemmas 1 and 2, we know all branches of $T$ must be of the type $B_{i}$ or $B_{i}^{*}, i=1,2, \ldots$. And from Theorem 4 and Lemmas 10 and 12, we know all branches of $T$ must be of the type $B_{i}$ or $B_{i}^{*}, i=2,3,4$.

Then the minimal ABC tree $T \in T_{n, 2}$ has

$$
n=1+5 N\left(B_{2}\right)+7 N\left(B_{3}\right)+9 N\left(B_{4}\right)+x
$$

vertices, where $x \in\{0,1\}$ counts the pendent paths of length 3.

From above we see that the tree $T \in T_{n, 2}$ with minimal $\mathrm{ABC}$ index and $x=0$ must possess the structure as shown in Figure 11(a). It is easy to see that

$\operatorname{ABC}(T)$

$$
\begin{aligned}
= & N\left(B_{2}\right) \sqrt{\frac{d_{u}+1}{3 d_{u}}}+N\left(B_{3}\right) \sqrt{\frac{d_{u}+2}{4 d_{u}}}+N\left(B_{4}\right) \sqrt{\frac{d_{u}+3}{5 d_{u}}} \\
& +\left(n-1-N\left(B_{2}\right)-N\left(B_{3}\right)-N\left(B_{4}\right)\right) \frac{\sqrt{2}}{2}
\end{aligned}
$$




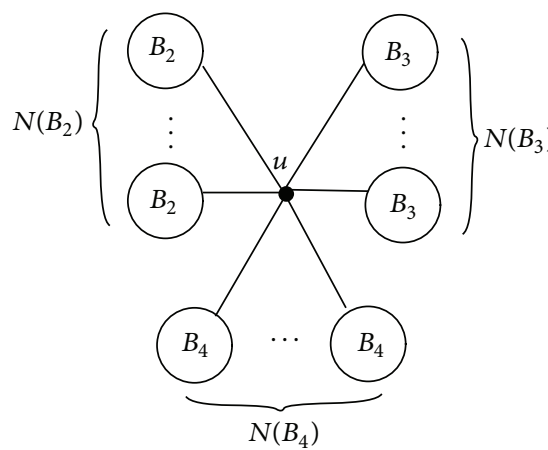

(a) The minimal $\mathrm{ABC}$ tree $T$ in $T_{n, 2}$

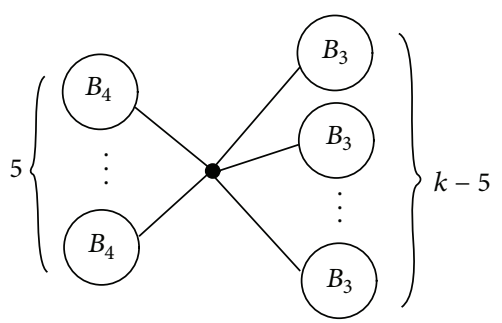

(b) $T_{4}{ }^{\prime}$

Figure 11

Putting (36) in the above equation, we have

$$
\begin{aligned}
f(D) \triangleq & \mathrm{ABC}(T) \\
= & N\left(B_{2}\right) \sqrt{\frac{d_{u}+1}{3 d_{u}}}+N\left(B_{3}\right) \sqrt{\frac{d_{u}+2}{4 d_{u}}} \\
& +N\left(B_{4}\right) \sqrt{\frac{d_{u}+3}{5 d_{u}}}+\left(4 N\left(B_{2}\right)+6 N\left(B_{3}\right)\right. \\
& \left.+8 N\left(B_{4}\right)+x\right) \frac{\sqrt{2}}{2},
\end{aligned}
$$

where $d_{u}=\Delta=N\left(B_{2}\right)+N\left(B_{3}\right)+N\left(B_{4}\right)$ and $D=$ $\left(N\left(B_{2}\right), N\left(B_{3}\right), N\left(B_{4}\right), x\right)$.

From Lemma 11 and Theorems 5 and 7, we get

$$
\begin{gathered}
N\left(B_{2}\right) \in\{0,1,2\}, N\left(B_{4}\right) \in\{0,1,2,3,4,5,6\}, \\
N\left(B_{2}\right) N\left(B_{4}\right)=0 .
\end{gathered}
$$

Note that the parameters $n, N\left(B_{2}\right), N\left(B_{3}\right), N\left(B_{4}\right)$, and $x$ in (36) are nonnegative integers.

\section{Consider}

(i) $n \equiv 0(\bmod 7)$ and $n=7 k+28$.

Case 1.1. If $N\left(B_{2}\right)=0$, then $n=1+7 N\left(B_{3}\right)+9 N\left(B_{4}\right)+x \equiv 0$ $(\bmod 7)$.

Thus, $9 N\left(B_{4}\right)+x+1 \equiv 0(\bmod 7)$, we get $N\left(B_{4}\right)=3$, $x=0$ or $N\left(B_{4}\right)=6, x=1$.

That is, $D=(0,(n / 7)-4,3,0)=(0, k, 3,0)$ or $(0,(n / 7)-$ $8,6,1)=(0, k-4,6,1)$.

Case 1.2. If $N\left(B_{2}\right)=1$, then $N\left(B_{4}\right)=0, n=1+5+7 N\left(B_{3}\right)+$ $x \equiv 0(\bmod 7)$; we get $x=1$.

That is, $D=(1,(n / 7)-1,0,1)=(1, k+3,0,1)$.

Case 1.3. If $N\left(B_{2}\right)=2$, then $N\left(B_{4}\right)=0$ and $n=11+7 N\left(B_{3}\right)+$ $x \equiv 0(\bmod 7)$; there is no solution.
Comparing the values $f(0, k, 3,0), f(0, k-4,6,1)$, and $f(1, k+3,0,1)$ and noting that $\Delta \geq 24$, we get that

$$
f(0, k, 3,0)=k \sqrt{\frac{k+5}{4(k+3)}}+3 \sqrt{\frac{k+6}{5(k+3)}}+(3 k+12) \sqrt{2}
$$

is the smallest one $(k \geq 21)$; the result (i) follows.

Consider

(ii) $n \equiv 1(\bmod 7)$ and $n=7 k+1$.

Case 2.1. If $N\left(B_{2}\right)=0$, then $9 N\left(B_{4}\right)+x+1 \equiv 1(\bmod 7)$.

We get $N\left(B_{4}\right)=0, x=0$ or $N\left(B_{4}\right)=3, x=1$.

That is, $D=(0, k, 0,0)$ or $(0, k-4,3,1)$.

Case 2.2. If $N\left(B_{2}\right)=1$, then $N\left(B_{4}\right)=0$ and $n=1+5+$ $7 N\left(B_{3}\right)+x$.

Thus $5+x \equiv 0(\bmod 7)$; there is no solution.

Case 2.3. If $N\left(B_{3}\right)=2$, then $N\left(B_{4}\right)=0$ and $n=1+10+$ $7 N\left(B_{3}\right)+x$.

Thus $10+x \equiv 0(\bmod 7)$; there is no solution.

Comparing the values $f(0, k, 0,0), f(0, k-4,3,1)$, we get that

$$
f(0, k, 0,0)=k \sqrt{\frac{k+2}{4 k}}+3 k \sqrt{2}
$$

is the smallest one $(k \geq 24)$; the result (ii) follows.

Consider

(iii) $n \equiv 2(\bmod 7)$ and $n=7 k+9$.

Case 3.1. If $N\left(B_{2}\right)=0$, then $n=1+7 N\left(B_{3}\right)+9 N\left(B_{4}\right)+x \equiv$ $2(\bmod 7)$.

We get $N\left(B_{4}\right)=0, x=1$ or $N\left(B_{4}\right)=4, x=0$.

That is, $D=(0, k+1,0,1)$ or $(0, k-4,4,0)$.

Case 3.2. If $N\left(B_{2}\right)=1$, then $N\left(B_{4}\right)=0$ and $n=1+5+$ $7 N\left(B_{3}\right)+x$.

Thus $6+x \equiv 2(\bmod 7)$; there is no solution.

Case 3.3. If $N\left(B_{2}\right)=2$, then $N\left(B_{4}\right)=0$ and $n=1+10+$ $7 N\left(B_{3}\right)+x$. 
Thus $11+x \equiv 2(\bmod 7)$; there is no solution.

Comparing the values $f(0, k+1,0,1), f(0, k-4,4,0)$, we get that

$$
f(0, k-4,4,0)=(k-4) \sqrt{\frac{k+2}{4 k}}+4 \sqrt{\frac{k+3}{5 k}}+(3 k+4) \sqrt{2}
$$

is the smallest one $(k \geq 168)$; the result (iii) follows.

Consider

(iv) $n \equiv 3(\bmod 7)$ and $n=7 k+10$.

Case 4.1. If $N\left(B_{2}\right)=0$, then $n=1+7 N\left(B_{3}\right)+9 N\left(B_{4}\right)+x \equiv$ $3(\bmod 7)$.

We get $N\left(B_{4}\right)=1, x=0$ or $N\left(B_{4}\right)=4, x=1$.

That is, $D=(0, k, 1,0)$ or $(0, k-4,4,1)$.

Case 4.2. If $N\left(B_{2}\right)=1$, then $N\left(B_{4}\right)=0$ and $n=1+5+$ $7 N\left(B_{3}\right)+x$.

Thus $6+x \equiv 3(\bmod 7)$; there is no solution.

Case 4.3. If $N\left(B_{2}\right)=2$, then $N\left(B_{4}\right)=0$ and $n=1+10+$ $7 N\left(B_{3}\right)+x$.

Thus $11+x \equiv 3(\bmod 7)$; there is no solution. that

Comparing the values $f(0, k, 1,0), f(0, k-4,4,1)$, we get

$$
f(0, k, 1,0)=k \sqrt{\frac{k+3}{4(k+1)}}+\sqrt{\frac{k+4}{5(k+1)}}+(3 k+4) \sqrt{2}
$$

is the smallest one $(k \geq 23)$; the result (iv) follows.

Consider

(v) $n \equiv 4(\bmod 7)$ and $n=7 k+11$.

Case 5.1. If $N\left(B_{2}\right)=0$, then $n=1+7 N\left(B_{3}\right)+9 N\left(B_{4}\right)+x \equiv$ $4(\bmod 7)$.

We get $N\left(B_{4}\right)=1, x=1$ or $N\left(B_{4}\right)=5, x=0$.

That is, $D=(0, k, 1,1)$ or $(0, k-5,5,0)$.

Case 5.2. If $N\left(B_{2}\right)=1$, then $N\left(B_{4}\right)=0, n=1+5+7 N\left(B_{3}\right)+x$.

Thus $6+x \equiv 4(\bmod 7)$; there is no solution.

Case 5.3. If $N\left(B_{2}\right)=2$, then $N\left(B_{4}\right)=0$ and $n=1+10+$ $7 N\left(B_{3}\right)+x$.

Thus $11+x \equiv 4(\bmod 7)$, we get $x=0$.

That is, $D=(2, k, 0,0)$.

Comparing the values $f(0, k, 1,1), f(0, k-5,5,0)$, and $f(2, k, 0,0)$, we get that

$$
f(2, k, 0,0)=k \sqrt{\frac{k+4}{4(k+2)}}+2 \sqrt{\frac{k+3}{3(k+2)}}+(3 k+4) \sqrt{2}
$$

is the smallest one $(k=22,23, \ldots, 286)$ and that

$$
f(0, k-5,5,0)=(k-5) \sqrt{\frac{k+2}{4 k}}+5 \sqrt{\frac{k+3}{5 k}}+(3 k+5) \sqrt{2}
$$

is the smallest one $(k \geq 287)$. The result (v) follows.

\section{Consider}

(vi) $n \equiv 5(\bmod 7)$ and $n=7 k+19$.

Case 6.1. If $N\left(B_{2}\right)=0$, then $n=1+7 N\left(B_{3}\right)+9 N\left(B_{4}\right)+x \equiv 5$ $(\bmod 7)$.

We get $N\left(B_{4}\right)=2, x=0$ or $N\left(B_{4}\right)=5, x=1$.

That is, $D=(0, k, 2,0)$ or $(0, k-4,5,1)$.

Case 6.2. If $N\left(B_{2}\right)=1$, then $N\left(B_{4}\right)=0$ and $n=1+5+$ $7 N\left(B_{3}\right)+x$.

Thus $6+x \equiv 5(\bmod 7)$; there is no solution.

Case 6.3. If $N\left(B_{2}\right)=2$, then $N\left(B_{4}\right)=0$ and $n=1+10+$ $7 N\left(B_{3}\right)+x$.

Thus $11+x \equiv 5(\bmod 7)$, we get $x=1$.

That is, $D=(2, k+1,0,1)$.

Comparing the values $f(0, k, 2,0), f(0, k-4,5,1)$, and $f(2, k+1,0,1)$, we get that

$$
f(0, k, 2,0)=k \sqrt{\frac{k+4}{4(k+2)}}+2 \sqrt{\frac{k+5}{5(k+2)}}+(3 k+8) \sqrt{2}
$$

is the smallest one $(k \geq 22)$; the result (vi) follows.

Consider

(vii) $n \equiv 6(\bmod 7)$ and $n=7 k+6$.

Case 7.1. If $N\left(B_{2}\right)=0$, then $n=1+7 N\left(B_{3}\right)+9 N\left(B_{4}\right)+x \equiv 6$ $(\bmod 7)$.

We get $N\left(B_{4}\right)=2, x=1$ or $N\left(B_{4}\right)=6, x=0$.

That is, $D=(0, k-2,2,1)$ or $(0, k-7,6,0)$.

Case 7.2. If $N\left(B_{2}\right)=1$, then $N\left(B_{4}\right)=0$ and $n=1+5+$ $7 N\left(B_{3}\right)+x$.

Thus $6+x \equiv 6(\bmod 7)$, we get $x=0$.

That is, $D=(1, k, 0,0)$.

Case 7.3. If $N\left(B_{2}\right)=2$, then $N\left(B_{4}\right)=0$ and $n=1+10+$ $7 N\left(B_{3}\right)+x$.

Thus $11+x \equiv 6(\bmod 7)$; there is no solution.

Comparing the values $f(0, k-2,2,1), f(0, k-7,6,0)$, and $f(1, k, 0,0)$, we get that

$$
f(1, k, 0,0)=k \sqrt{\frac{k+3}{4(k+1)}}+\sqrt{\frac{k+2}{3(k+1)}}+(3 k+2) \sqrt{2}
$$

is the smallest one $(k \geq 23)$; the result (vii) follows.

In [13], the authors also gave a similar result as Theorem 13 (in [13], a tree $T \in T_{n, 2}$ without pendent path of length 3 is called a proper Krag tree), but we do it independently and the methods are also different.

\section{Conflict of Interests}

The authors declare that there is no conflict of interests regarding the publication of this paper. 


\section{Acknowledgments}

The project was supported by the National Nature Science Foundation of China (nos. 11101087 and 11301440), by the Nature Science Foundation of Fujian Province (no 2013J05006), and by the Foundation to the Educational Committee of Fujian (nos. JA13025 and JA13034).

\section{References}

[1] E. Estrada, L. Torres, L. Rodríguez, and I. Gutman, "An atombond connectivity index: modelling the enthalpy of formation of alkanes," Indian Journal of Chemistry A, vol. 37, no. 10, pp. 849-855, 1998.

[2] E. Estrada, "Atom-bond connectivity and the energetic of branched alkanes," Chemical Physics Letters, vol. 463, no. 4-6, pp. 422-425, 2008.

[3] B. Furtula, A. Graovac, and D. Vukičević, "Atom-bond connectivity index of trees," Discrete Applied Mathematics, vol. 157, no. 13, pp. 2828-2835, 2009.

[4] B. Zhou and R. Xing, "On atom-bond connectivity index," Zeitschrift fur Naturforschung A, vol. 66, no. 1-2, pp. 61-66, 2011.

[5] J. Chen and X. Guo, "Extreme atom-bond connectivity index of graphs," MATCH Communications in Mathematical and in Computer Chemistry, vol. 65, no. 3, pp. 713-722, 2011.

[6] I. Gutman, B. Furtula, and M. Ivanović, "Notes on trees with minimal atom-bond connectivity index," MATCH Communications in Mathematical and in Computer Chemistry, vol. 67, no. 2, pp. 467-482, 2012.

[7] W. Lin, X. Lin, T. Gao, and X. Wu, "Proving a conjecture of Gutman concerning trees with minimal $A B C$ index," MATCH Communications in Mathematical and in Computer Chemistry, vol. 69, no. 3, pp. 549-557, 2013.

[8] I. Gutman and B. Furtula, "Trees with smallest atom-bond connectivity index," MATCH Communications in Mathematical and in Computer Chemistry, vol. 68, no. 1, pp. 131-136, 2012.

[9] D. Dimitrov, "Efficient computation of trees with minimal atom-bond connectivity index," Applied Mathematics AJournal of Chinese Universitiesnd Computation, vol. 224, pp. 663-670, 2013.

[10] J.-S. Chen and X.-F. Guo, "The atom-bond connectivity index of chemical bicyclic graphs," Applied Mathematics-A Journal of Chinese Universities, vol. 27, no. 2, pp. 243-252, 2012.

[11] J. Chen, J. Liu, and X. Guo, "Some upper bounds for the atom-bond connectivity index of graphs," Applied Mathematics Letters, vol. 25, no. 7, pp. 1077-1081, 2012.

[12] J. Chen, J. Liu, and Q. Li, "The atom-bond connectivity index of catacondensed polyomino graphs," Discrete Dynamics in Nature and Society, vol. 2013, Article ID 598517, 7 pages, 2013.

[13] S. A. Hosseini, M. B. Ahmadi, and I. Gutman, "Kragujevac trees with minimal atom-bond connectivity index," MATCH Communications in Mathematical and in Computer Chemistry, vol. 71, no. 1, pp. 5-20, 2014. 


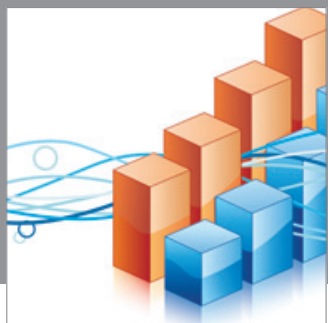

Advances in

Operations Research

mansans

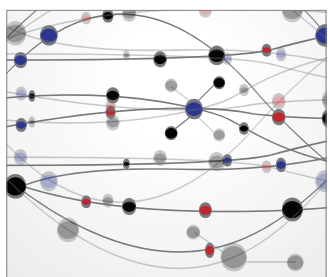

The Scientific World Journal
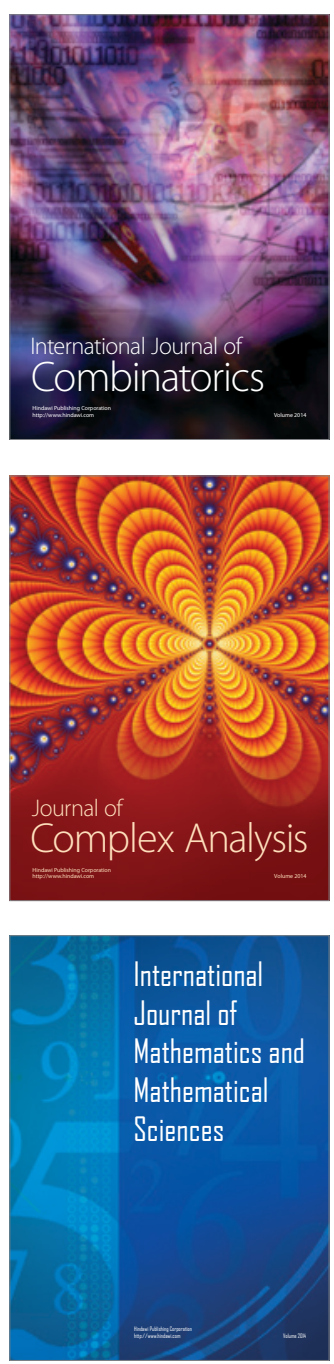
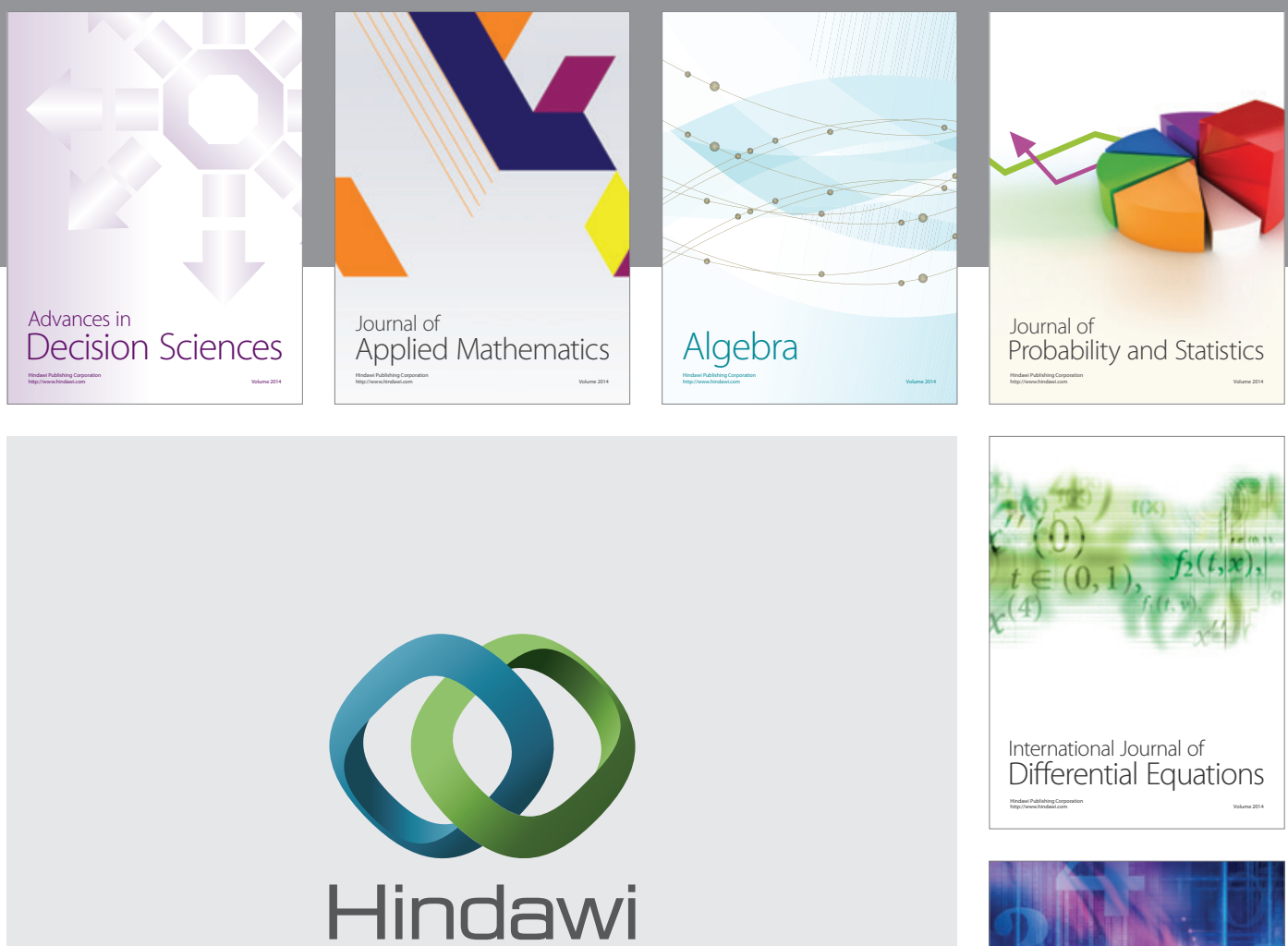

Submit your manuscripts at http://www.hindawi.com
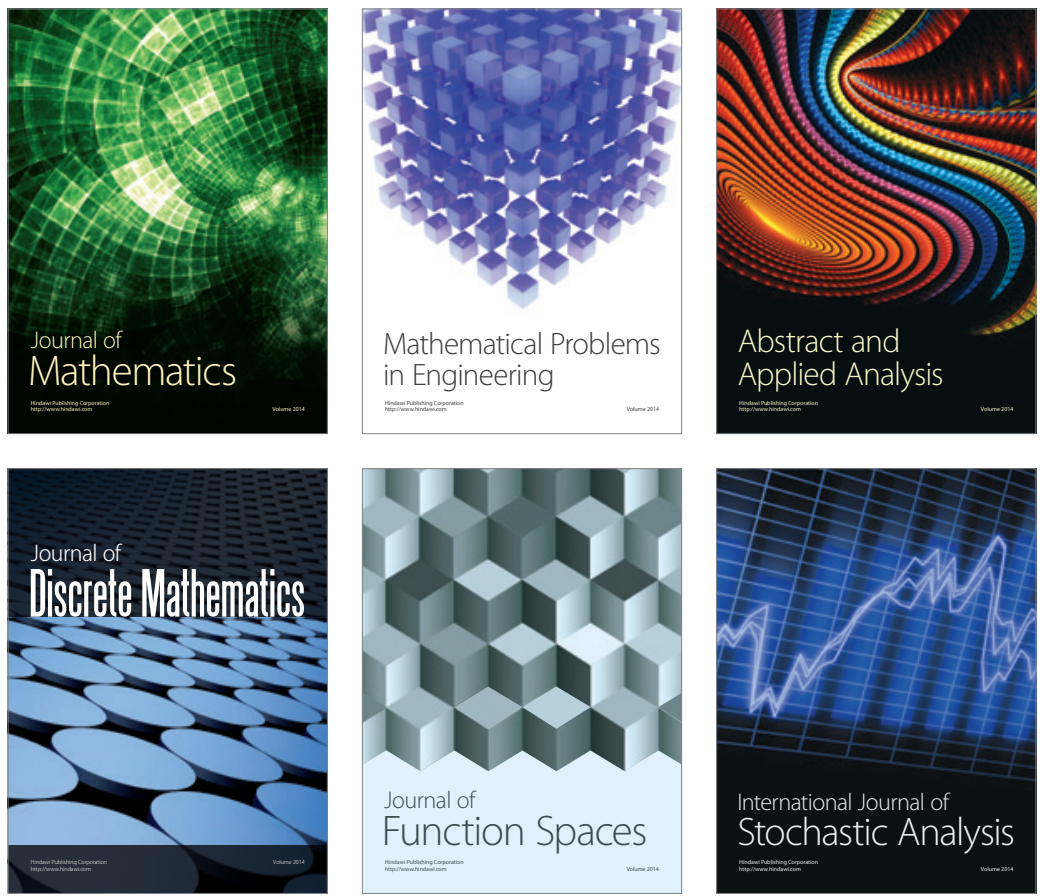

Journal of

Function Spaces

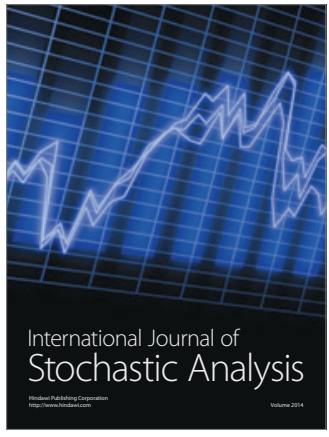

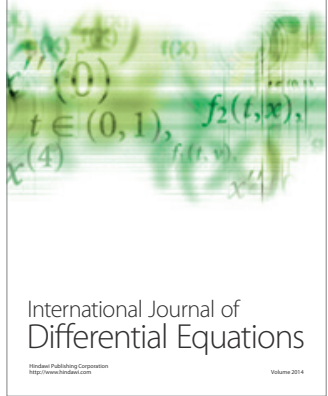
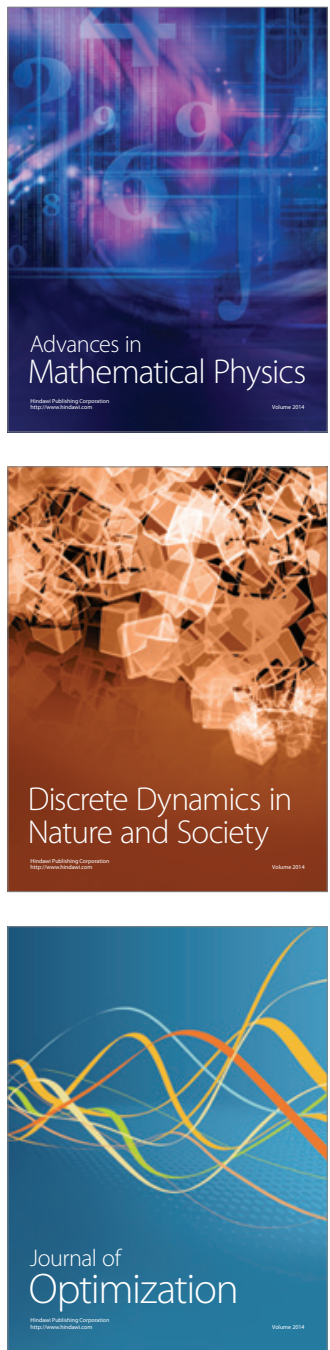